\title{
10 \\ Take home messages
}

Pedro Encarnação, Sylvie Ray-Kaeser, Nicole Bianquin and Serenella Besio

Below you can find a list of key ideas about play for children with disabilities that we would like you to reflect on upon reading this book:

- 'Play is a range of voluntary, intrinsically motivated activities associated with recreational pleasure and enjoyment'.

- Traits of play include being a child-lead activity, freely chosen, with no other goals besides the fun of playing.

- Playful activities with educational or therapeutic goals, although being an invaluable means to engage children, are not play but play-like activities.

- Play for the sake of play supports all aspects of a child's development and well-being.

- Play is a right for all children enshrined in international conventions such as the United Nations Convention on the Rights of the Child or the United Nations Convention on the Rights of Persons with Disabilities.

- Disability does not lie solely in the person, it is the result of a complex interaction between a person, with his/her health condition, abilities and personal factors, and the environment, including the physical, social, cultural and institutional contexts. Disability is the outcome of a misfit between a person and an environment with barriers (or without proper facilitators), restricting individual's activities and participation.

- Children with disabilities have the same desire to play as any other children. Play is equally important for their development and well-being. Moreover, play is the natural way for them to interact with peers, thus fostering inclusion. 
- Children with disabilities face many barriers to play, including

- institutional barriers (the importance of play is recognized by all in theory, but there's a lack of active play promotion policies);

- social-cultural barriers (play often occupies a secondary role when compared to educational or therapeutic activities, sometimes children with disabilities are ostracized of feel stigmatized); and

- physical barriers (lack of accessible playspaces, toys, games).

- Direct observation is the best way of assessing children's play. There are several questionnaires and observation grids that can support play assessment. This assessment is instrumental in planning proper interventions such that a child can fully enjoy his/her right to play for the sake of play.

- Play can be learned and taught.

- Adults can guide and support children with disabilities to play. It is fundamental that adults keep in mind what is play ensuring that their interventions do not jeopardize the children's play experience. The metaphor of scaffolding is particularly illustrative: adults should provide the support that is needed but, once children acquire the skills needed to play, adults should step down and allow children to take full control of the play.

- Play often requires staying in a comfortable and functional position, moving around, manipulating objects, communicating, or using a computer or tablet. There is a vast number of assistive products that may reduce the gap between the requirements of the play and the children's abilities by providing support in the above general-purpose functional areas or by adapting the activity.

- The secret of keeping a child engaged in play is providing the just-right amount of challenge. Too much challenge leads to frustration; too little challenge leads to boredom.

- There are no toys, games, playspaces suitable for every child and family. Each child and each family is unique, with his or her own preferences and interests. There are, however, general principles that can help enabling play for everyone. 
- Play is not an attribute of toys and games; play belongs to the players. Although toys and games are tools for play, children can play with whatever is available. They certainly don't need expensive toys in order to play.

- Toys and games should be accessible to the widest range of abilities possible; they should be designed in order to match the motor, sensory, perceptual, cognitive, socio-emotional and psychological skills of the broadest possible group of children (not too easy, not too complicated, the just-right amount of challenge).

- Children nowadays are usually attracted by electronic devices and digital games. Although there is the need of ensuring enough opportunities for more physically and socially demanding play activities, children should not be prohibited from playing with digital games as it will develop skills they will need to live in our digital world.

- It is not all about the toy and the game. Although play can happen anywhere, a dedicated space and time for play, with the right toys and (if needed) the right assistive products, the right playmates and the right play context can better enable the child to play.

- The play value of a space refers to the amount of play opportunities available that the children can access or use, and wish to use.

- A playground with high play value, that provides the just-right amount of challenge for every child, will have some components that are not usable by all children. A rule of thumb might be: a playground should be $100 \%$ welcoming, $70 \%$ accessible and 50\% usable.

- Play in natural environments should also be accessible to children with disabilities.

- It will only be play if the child is in control and feels involved. Make sure to properly assess the particular preferences and interests of a child, and seek the child's opinion. The experts on play are the children!

- A new culture of play for all, especially for children with disabilities, is needed. Societies should internalize the invaluable importance of play for the sake of play and strive for policies that ensure that all children can enjoy their right to play. 